\title{
Assessing the prognostic value of PAX2 and PTEN in endometrial carcinogenesis
}

\author{
Emma Rewcastle1,*, Anne Elin Varhaugvik1,2,*, Einar Gudlaugsson', Anita Steinbakk1,3, Ivar Skaland', \\ Bianca van Diermen', Jan P Baak ${ }^{1,4, *}$ and Emiel A M Janssen'1,5,* \\ 'Department of Pathology, Stavanger University Hospital, Stavanger, Norway \\ 2Department of Pathology, Helse Møre og Romsdal, Ålesund, Norway \\ ${ }^{3}$ Stavanger-Gynekologene AS, Stavanger, Norway \\ ${ }^{4}$ Dr. Med. Jan Baak AS, Tananger, Norway \\ ${ }^{5}$ Department of Mathematics and Natural Sciences, University of Stavanger, Stavanger, Norway
}

Correspondence should be addressed to J P Baak: drmedjanbaakas@yahoo.com

*(E Rewcastle, A E Varhaugvik, J P Baak and E A M Janssen contributed equally to this work)

\begin{abstract}
In order to avoid the consequences of over- and under-treatment of endometrial hyperplasia, diagnostic accuracy and progression risk assessment must be improved. The aim of this study was to assess whether PAX2 or PTEN expression could predict progression-free survival in endometrial intraepithelial neoplasia (EIN) and endometrial endometrioid carcinoma (EEC). Immunohistochemistry for detection of PAX2 and PTEN was performed on 348 endometrial samples; 75 proliferative endometrium (PE), 36 EIN and 237 EEC. Cases classified as PTEN null (1 or more glands negatively stained) were more prevalent in EEC than in PE and EIN (64\% EEC vs $11 \%$ PE/EIN). A progressive decrease in PAX2 expression was observed from PE to EIN to EEC. Long-term clinical follow-up (6-310 months, median: 126) was available for 62 PE cases, all 36 EIN cases and 178 EEC cases. No patients with PE demonstrated progression to EIN or EEC. Progression of disease was observed in $10(28 \%)$ EIN patients. These patients had significantly lower PAX2 expression than those that regressed $(P=0.005)$. Progression-free survival analysis revealed that EIN patients with a high-risk PAX2 expression score (H-score $\leq 75)$ had a higher probability of progression of disease in comparison to those with a low-risk score (H-score $>75)$. PAX2 expression was not prognostic in EEC nor was PTEN status of prognostic value in either EIN or EEC. PAX2 expression analysis by means of $\mathrm{H}$-score has prognostic potential for the identification of high-risk progression cases in EIN but needs to be validated in a larger cohort.
\end{abstract}

\author{
Key Words \\ - endometrial endometrioid \\ carcinoma \\ - endometrial intraepithelial \\ neoplasia (EIN) \\ - PAX2 \\ - PTEN \\ - prognostic biomarkers
}

Endocrine-Related Cancer (2018) 25, 981-991

\section{Introduction}

Endometrial cancer is currently the most frequent form of gynaecologic cancer in developed countries (http:// globocan.iarc.fr/Pages/fact_sheets_population.aspx, Accessed: 28 February 2018). Endometrial endometrioid cancer (EEC) is often preceded by a premalignant lesion termed endometrial hyperplasia (Bokhman 1983). These lesions have been previously categorised by the WHO94 system according to the degree of glandular complexity and nuclear atypia; however, the disadvantages of its use in the clinic has been greatly debated citing its poor 
reproducibility and inadequate predictive capability (Skov et al. 1997, Kendall et al. 1998, Bergeron et al. 1999). The newer EIN classification system, recently adopted by the World Health Organisation (WHO) in its 2014 publication (Kurman et al. 2014), demonstrates improved reproducibility and clinical predictive ability compared to the older scheme (Baak et al. 2005a, Hecht et al. 2005, Mutter \& Group TIENW 2000, Usubutun et al. 2012). The EIN classification distinguishes two forms of endometrial hyperplasia: benign reactive hyperplasia, which is a polyclonal, diffuse lesion that is a result of hormone imbalance and oestrogen hyper-stimulation, and endometrial intraepithelial neoplasia (EIN), defined as a monoclonal, neoplastic lesion that has a high risk of progression to EEC (Mutter \& Group TIENW 2000). Of note, the EIN system more clearly distinguishes high progression risk categories from low risk compared to the WHO94 system, especially when EIN is diagnosed by computerised morphometric analysis or D-score (Baak et al. 2005a).

With the ability to better distinguish between highrisk and low-risk lesions; a clearer prognosis can result in improved allocation of the most appropriate treatment. Despite an EIN diagnosis predisposing to an increased risk of EEC, some patients will never develop EEC, and likewise some rare non-EIN cases can develop EEC. For premenopausal women wishing to preserve fertility and high-risk surgery patients, hysterectomy may be considered as over-treatment. However, as the chances of concurrent carcinoma is high and D-score diagnosed EIN are reported to have a 45 -fold risk of progressing to EEC (Baak etal.2005a, Kurman etal.2014), progestin treatments and monitoring may result in under-treatment. It would therefore be beneficial to be able to ascertain which cases are likely to progress to reduce the likelihood of over and under-treatment, particularly for these patients. Currently, the EIN classification alone is unable to $100 \%$ accurately predict which endometrial cases will progress to cancer. To achieve optimal management of patients, discrimination between lesions that should be handled differently is crucial. There is therefore precedence for the establishment of accurate prognosticators to distinguish between low and high progression risk EIN.

Considerable research has been conducted searching for prognostic biomarkers to predict progression amongst EIN and EEC cases. One of the most commonly investigated biomarkers implicated in endometrial carcinogenesis is PTEN and of recent interest PAX2.
The tumour suppressor gene PTEN (10q23.31) is found inactivated in up to $83 \%$ of EEC (Mutter et al. 2000). It encodes the PTEN protein, a lipid phosphatase which functions as a negative regulator of the PI3K/AKT pathway (Kurose et al. 2001). The PAX2 gene (10q24.31) encodes PAX2 (paired-box protein 2), a transcription factor active in embryogenesis and promotion of cell proliferation (Shang 2007). Oestrogen- and tamoxifeninduced hypomethylation of the PAX2 promoter has been demonstrated in endometrial carcinogenesis ( $\mathrm{Wu}$ et al. 2005). Several immunohistochemical studies have shown that loss of expression of both PTEN and PAX2 increases from normal endometrium to endometrial hyperplasia to EEC (Mutter et al. 2000, Baak et al. 2005b, Monte et al. 2010, Pavlakis et al. 2010, Allison et al. 2012). Furthermore, combined loss of PTEN and PAX2 in endometrial glands has been described as an early event and is suggested to promote neoplastic transformation, although they are activated independently (Mutter $\mathrm{et}$ al. 2000, Monte et al. 2010, Allison et al. 2012). Notably, in one study of 103 hyperplasias, no morphometrically high-risk (i.e., D-score <1) EIN lesions with PTEN-positive glands developed cancer in a long follow-up, contrasting to a high cancer incidence in PTEN negative, D-score $<1$ cases (Baak et al. 2005b). Thus, in EIN, D-score and PTEN might have additional prognostic value. This suggests a combinatorial approach to deduce prognosis could be promising. PAX2 expression has been reported to be diminished in EEC (Strissel et al. 2008, Monte et al. 2010, Allison et al. 2012) but one study has demonstrated increased expression in EEC (Kahraman et al. 2012). No studies have, to the best of our knowledge, yet commented on whether PAX2 is associated with progression-free survival in EEC. Therefore, a thorough prognostic evaluation of the additive prognostic value of PAX2 would be beneficial.

In our investigation, we used strictly optimised and standardized immunohistochemistry to observe expression patterns of PTEN and PAX2 in proliferative endometrium (PE), EIN and EEC. We aimed to assess whether PAX2 and PTEN expression has the potential to predict progression-free survival in a large EEC study population with long-term follow-up, in addition to its potential to predict progression of EIN to EEC. We aimed to review their potential as prognostic biomarkers in order to improve low-risk and high-risk progression categorisation so as to avoid the risks of over- and undertreatment of EIN and EEC. 


\section{Materials and methods}

\section{Study population}

This study was approved by the Regional Ethics Committee of Health West Norway (2010/2464). None of the patients included in this study were required to provide written informed consent to participate. All insights in a patient's journal were monitored electronically, and all, except the treating physician, were required to state the reason why they needed to read that patient's journal. This log was always open for the patient to view. A total of 348 cases were selected from a vast database, which includes 5494 patients each with one or more endometrial histology samples from the archives of the Department of Pathology, Stavanger University Hospital, Stavanger, Norway, dating from 1977 to 2004 (Steinbakk et al. 2009). Cases were carefully and independently reviewed by two pathologists (J B, E G) using strict microscopic criteria and classified as PE, EIN or endometrioid type endometrial carcinoma (EEC). In case of discrepancies in diagnosis between the pathologists, a consensus meeting with a multi-head microscope was used to decide on a final diagnosis. In total, 75 cases with the diagnosis PE, 36 cases with EIN and 237 with ECC were included in the current study (Table 1). Formalin-fixed paraffin-embedded pipelle/curettage/hysterectomy samples were available for all these cases. All endometrial carcinoma samples were available as tissue microarrays (TMA) created as described by Steinbakk et al. (2009). Histologic sections $4 \mu \mathrm{m}$ thick underwent standard haematoxylin and eosin (H\&E) staining. All endometrial cancer samples were classified according to FIGO stage (Table 1) whilst all EIN samples were classified according to WHO14 guidelines. Inclusion criteria for retrospective analysis required follow-up of at least 6 months. Follow-up data were available for 62 of the $75 \mathrm{PE}$ cases (median: 131, range: 6-202 months), all 36 EIN cases (median: 138, range: 13-275) and 178 EEC cases (median: 128, range: 6-310).

\section{Immunohistochemistry}

The protocols for H\&Estaining and immunohistochemistry were performed as outlined previously (Baak et al. 2005b).
Histologic sections were cut to $4 \mu \mathrm{m}$ adjacent to $\mathrm{H} \& \mathrm{E}$ sections. Sections were mounted on silanised slides (Dako) and dried overnight at $37^{\circ} \mathrm{C}$. The slides were incubated at $60^{\circ} \mathrm{C}$ for $1 \mathrm{~h}$ and then deparaffinised in xylene followed by rehydration in decreasing concentrations of alcohol. For PAX2 and PTEN, antigen retrieval was performed in the following conditions: $120^{\circ} \mathrm{C}, 1.9$ bar in $10 \mathrm{mmol} / \mathrm{L}$ TRIS/1 mmol/L EDTA (pH 9.0) for 2 min then cooled for $15 \mathrm{~min}$. Immunostaining was performed using an automated stainer from Dako with Tris-buffered saline (S1968) 0.05\% Tween 20 (pH 7.6) as a wash buffer. Endogenous peroxidase activity was blocked with a peroxidase blocking reagent (S2001, Dako) for $10 \mathrm{~min}$. The slides were then incubated for $30 \mathrm{~min}$ with a PTEN monoclonal antibody (1:300, clone 6h2.1, Cascade Biosciences, Winchester, MA, USA) or PAX2 polyclonal antibody (1:200, cat. No 71-60000, Invitrogen). A peroxidase/DAB system (ChemMate Envision Kit, Dako) was used to visualise the immune-complex; slides were incubated in Envision/HRP secondary antibodies for $30 \mathrm{~min}$ and thereafter $10 \mathrm{~min}$ in DAB-chromogen. Sections were then counterstained with haematoxylin, dehydrated and mounted. Immunostaining of adjacent normal endometrial tissue and positive immunostaining of endometrial stroma, for PTEN, were considered as internal controls.

\section{Evaluation of immunohistochemical results}

For each sample with successful staining a single area, between $2 \times 2 \mathrm{~mm}$ and $5 \times 5 \mathrm{~mm}$ in size, was selected for evaluation of PTEN and PAX2. For cases diagnosed as PE, a representative area was chosen by one of the pathologists (J B, E G) and scored by two independent observers (A E V, E J). For cases diagnosed with EIN, an experienced pathologist (J B) marked an area of the section with the most high-graded lesion for evaluation. For endometrioid endometrial cancer (EEC) samples, the TMA's contained the most poorly differentiated, well-preserved tumour areas and therefore each individual TMA was considered as a single measurable area. Two samples were taken from each EEC case for TMA and their mean score was used

Table 1 Overview of the number of cases in each diagnostic category with successful PTEN and PAX2 staining.

\begin{tabular}{|c|c|c|c|c|c|c|c|c|c|c|c|c|}
\hline & \multirow[b]{2}{*}{ PE* $(N=75)$} & \multirow[b]{2}{*}{$\mathbf{E I N}^{+}(N=36)$} & \multicolumn{10}{|c|}{ EEC ${ }^{\prime}(\mathrm{FIGO}$ stage $)(N=237)$} \\
\hline & & & la & $\mathrm{Ib}$ & IC & Ila & Ilb & IIla & IIIb & IIIC & IV & Total \\
\hline \multicolumn{13}{|c|}{ Number of cases with successful staining according to diagnostic category } \\
\hline PTEN & $71 / 75$ & $35 / 36$ & 23/31 & $86 / 122$ & $35 / 39$ & $8 / 9$ & $11 / 14$ & $8 / 9$ & $0 / 1$ & $5 / 6$ & $1 / 6$ & $177 / 237$ \\
\hline PAX2 & $75 / 75$ & $36 / 36$ & $25 / 31$ & $89 / 122$ & $36 / 39$ & $8 / 9$ & $11 / 14$ & $8 / 9$ & $0 / 1$ & $5 / 6$ & $1 / 6$ & $183 / 237$ \\
\hline
\end{tabular}

*Proliferative endometrium; †'endomterial intraepithelial neoplasia; ${ }^{\ddagger}$ endometrioid endometrial carcinoma. 
for analysis. Two trained observers (A E V, E J), blinded to the outcome, independently evaluated and scored each case using a light microscope (objective 40x, field diameter $450 \mu \mathrm{m}$ at specimen level). PTEN staining was determined as positive or negative by presence or absence of nuclear or cytoplasmic staining respectively; stromal PTEN staining was used as a positive control. Sections containing one or more PTEN-null glands within the marked area were scored as negative (J B), as previously described (Steinbakk et al. 2009). Sections stained with PAX2 were scored using the H-score method. Glandular nuclei could be assigned a score of $0-3$ according to staining intensity $(0=$ negative, 1 =weak positive, $2=$ positive, $3=$ strongly positive). The observers would evaluate the marked area and deduce the percentage of glandular nuclei at each staining level. Each staining score was then multiplied with the respective percentage, and these were then added together to result in an $\mathrm{H}$-score that ranged from 0 to 300. If PAX2 H-score between the two observers differed by more than 50 a consensus meeting with a multi-head microscope was used to decide on a final score. If the PAX2 score differed by less than 50 points the average of the two scores determined the final score.

\section{Computerised image analysis}

The computerised morphometric analysis termed D-score was performed, as previously described (Baak et al. 2001), on H\&E-stained sections using the QPRODIT system (Leica). Lesions scored as D-score $<1$ were defined as highrisk for progression whilst those scored as D-score $\geq 1$ were deemed as low risk. D-score was performed on 35 of 36 EIN cases, where one lesion did not meet the size criteria.

\section{Statistical analysis}

Descriptive statistics and cross tables were used to control database quality, to identify any unlikely values or combinations. Regression (no change in diagnosis for PE cases) or progression of endometrial disease was used as the measured endpoints. Regression for EIN and EEC was defined as cured, no recurrence at final follow-up. For cases with an initial diagnosis of $\mathrm{PE}$, progression was defined by a diagnosis of EIN, EEC or EEC-related death in the follow-up. For cases with an initial diagnosis of EIN, progression was defined by a diagnosis of EEC or EEC-related death in the follow-up. For the purposes of this study, EIN cases that persisted in the follow-up, with a lower D-score compared to the original sample, were grouped together with progression cases. For cases with an initial diagnosis of EEC, progression was defined by a diagnosis of higher stage disease or EEC-related death in the follow-up; patients diagnosed with higher stage disease gave the same results as those who succumbed to EEC and were therefore grouped together in this study. Patients who were either lost to follow-up or suffered death from other non-EEC-related causes were censored to last known follow-up date as alive, no evidence of disease. SPSS (SPSS) for Windows, version 23.0 was used for all analyses. A one-way ANOVA was used to observe differences between the three initial diagnostic categories, and an independent sample $t$-test was used to compare progression and regression patients within each category, according to age, follow-up and PAX2. A chi-square test was used to test for significant differences between progression and regression patients within each category. For survival analysis, Kaplan-Meier survival curves were created and significant differences between groups were tested using the log-rank test. The endpoint was progression-free survival. Data were considered significant at $P<0.05$.

\section{Results}

Age distribution of patients varied between the groups. For patients diagnosed with PE the mean age was 38 (range 25-50), for EIN 46 years (range 24-87) and for EEC 66 years (range 32-92) (Table 2). The lesion of interest was lost in one case for EIN and four cases for PE for PTEN staining (Table 1). Of the 237 EEC samples, PAX2 and

Table 2 Mean age, mean PAX2 score and PTEN status according to endometrial diagnostic category.

\begin{tabular}{|c|c|c|c|}
\hline & PE* & EIN $^{+}$ & EEC $¥$ \\
\hline$N$ & 75 & 36 & 183 \\
\hline Mean age (range) & $38(25-50)^{\S}$ & $46(24-87)^{\S}$ & $66(32-92) \S$ \\
\hline Mean PAX2 score (range) & $157(0-245)^{\S}$ & $123(0-300) \S$ & $55(0-215)^{\S}$ \\
\hline PTEN \% cases & $\begin{array}{c}89 \% \text { pos }(63 / 71) \\
11 \% \text { neg }(8 / 71)\end{array}$ & $\begin{array}{c}89 \% \text { pos }(31 / 35) \\
11 \% \text { neg }(4 / 35)\end{array}$ & $\begin{array}{c}34 \% \text { pos }(61 / 177) \\
66 \% \text { neg }(116 / 177)\end{array}$ \\
\hline
\end{tabular}

*Proliferative endometrium; ${ }^{\dagger}$ endometrial intraepithelial neoplasia; ${ }^{\ddagger}$ endometrioid endometrial carcinoma; ${ }^{\S}$ significant between all groups: One-Way ANOVA, $P<0.05$. 
PTEN staining was successful in $183(183 / 237,77.2 \%)$ and 177 (177/237, 74.7\%) cases, respectively (Table 1).

Endometrial samples demonstrating a loss of PTEN glandular expression could be readily identified; glands with no PTEN staining were distinct from glands with strong PTEN staining and surrounding stroma (Fig. 1). In PE and EIN, 89\% were categorised as PTEN positive and $11 \%$ were PTEN negative (Table 2). Whereas in EEC, 34\% were classified as PTEN-positive and $66 \%$ as PTEN negative (Table 2). No difference in PTEN status was observed between different FIGO stages of EEC (data not shown).

Whilst staining for PTEN allowed for distinct definition of PTEN-null glands, PAX2 exhibited a nuclear staining pattern with variable intensity (Fig. 1). A decreasing trend in PAX2 expression was observed with increasing severity of diagnosis where mean scores decreased from 157 for PE to 123 for EIN to 55 for EEC, which was found to be significant (PE vs EIN: $P=0.017$, EIN vs EEC: $P<0.001$, PE vs EEC: $P<0.001$ ) (Fig. 2 and Table 2). There was no observable difference in PAX2 expression between FIGO stages of EEC (data not shown). Scoring of PAX2 had good reproducibility, where $22.4 \%$ (41/183) of EEC cases demonstrated conflict between the two observers and a consensus discussion was required.

For 62 of 75 PE cases with known follow-up, none demonstrated progression. Follow-up criteria were met for all 36 patients with an initial EIN diagnosis whilst 178 EEC patients met follow-up criteria. Of the 178 EEC patients with follow-up, all had successful PAX2 staining and 172 had successful PTEN staining.

Of the $36 \mathrm{EIN}$ patients, 26 demonstrated regression of disease, whilst seven patients were diagnosed with EEC. The remaining three patients were diagnosed as persistent EIN at final follow-up. Of note, although still diagnosed with EIN in the follow-up, all three patients had a lower D-score in the follow-up sample in addition to the observation that the glands were more tightly packed and crowded with less overall stroma over a wider area in direct comparison to the original lesion. As a result, these patients were grouped together with the seven progression cases in this study and henceforth these ten patients will be referred to as progression cases. These ten patients were found to have a significantly lower average PAX2 expression score in the original EIN sample in comparison to patients that regressed $(P=0.005)$ (Fig. 3). In EIN progression cases, $40 \%$ were PTEN negative in the initial sample, whereas $20 \%$ of EIN regression cases were PTEN negative; however, this difference was not significant (Table 3). For patients with an initial diagnosis of EEC, no difference was observed between average PAX2 expression scores or PTEN status of patients with or without progression (Table 3). EIN progression risk scores by the D-score method were calculated for 35 of 36 EIN cases. Patients with an initial diagnosis of EIN who later progressed had a significantly lower D-score than cases that demonstrated regression $(P=0.013)$ (Table 3$)$.
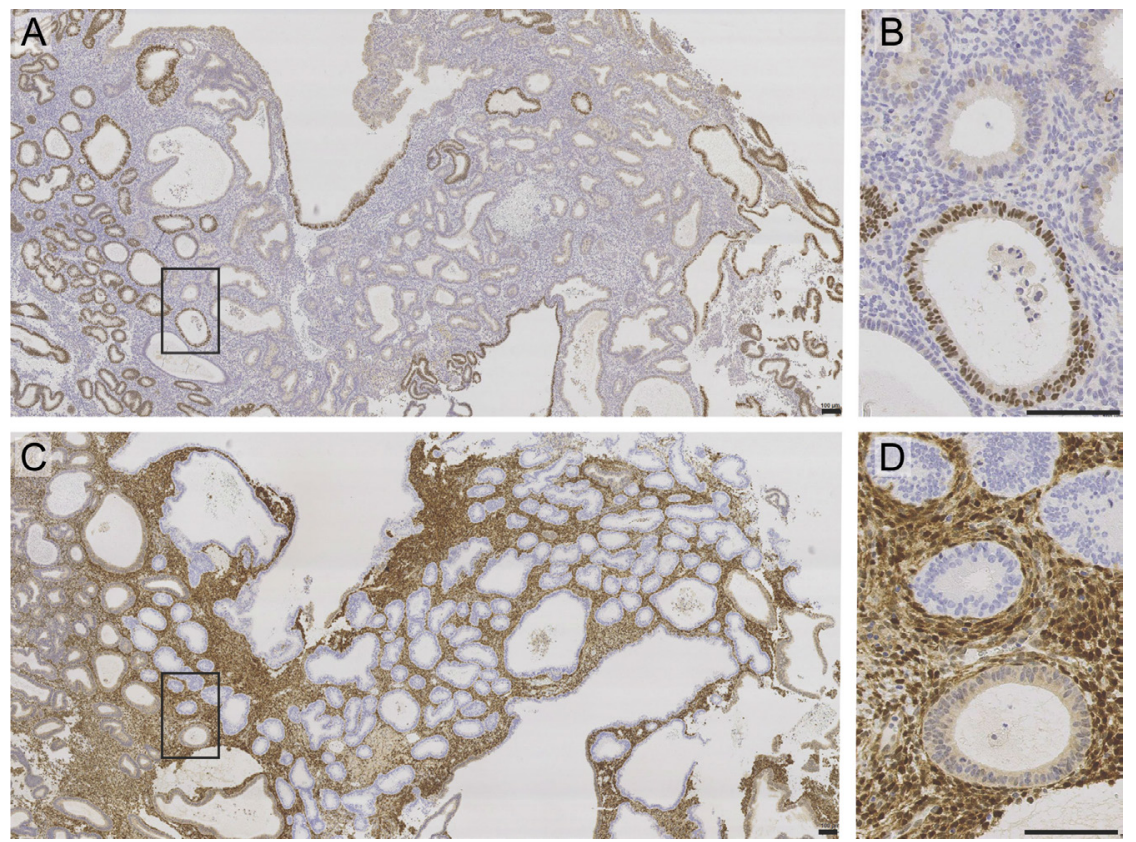

Figure 1

Example of an EIN lesion immunostained for the detection of (A and B) PAX2 or (C and D) PTEN protein. The $D$-score was -0.5 . (A) Intensity of PAX2 staining was observed to vary across the lesion. Of note, glands with weak expression and glands with strong expression tended to group within the lesion. (B) A close-up of glands indicated by the box in (A) compares a gland with low expression (top) with a gland with high PAX2 expression (bottom). PAX2 expression could also vary within a single gland with some nuclei strongly expressing PAX2 neighbouring nuclei that did not. (C) This lesion was defined as PTEN-null due to the presence of one or more PTEN-null glands (null glands indicated by absence of PTEN staining). PTEN-negative staining was very distinct from PTEN-positive staining with neighbouring glands showing contrasting expression in some areas. (D) A close-up of the glands indicated by the box in (C) emphasising that PTEN staining was very distinct readily distinguishing PTEN-positive (bottom) glands from PTEN-negative glands (top). Scale bar represents $100 \mu \mathrm{m}$. A full colour version of this figure is available at https://doi.org/10.1530/ ERC-18-0106. Printed in Great Britain 


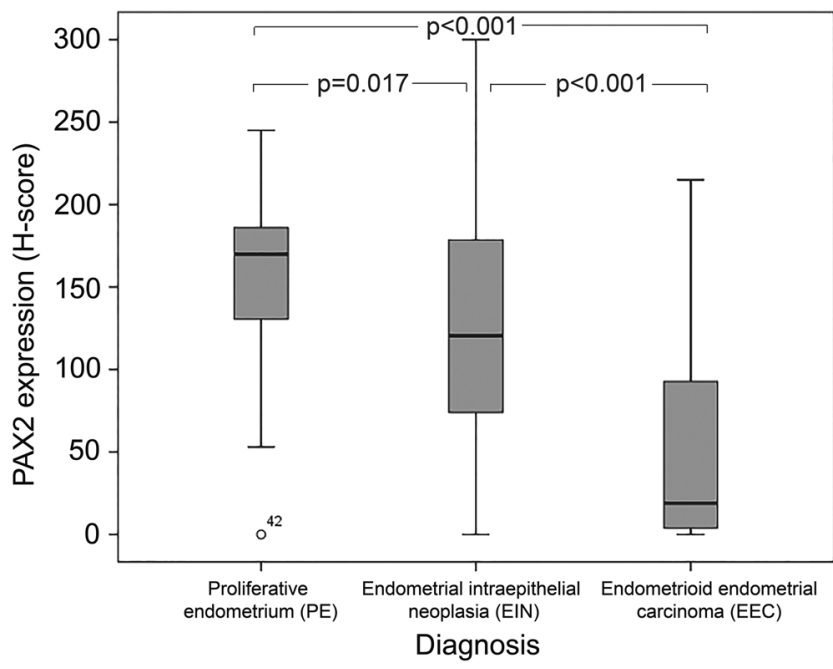

Figure 2

PAX2 expression according to diagnosis. All diagnostic categories had significantly different PAX2 expression scores (one-way ANOVA, $P<0.05$ ).

Age at the time of initial diagnosis was also evaluated for any potential differences between progression and regression cases in EIN and EEC. Only for the EIN category was a significant difference observed between the age of patients that demonstrated regression (mean 42 years) compared to progression (mean 57 years) $(P=0.029)$ (Table 3).

Expanding on the observation that EIN progression patients had a lower mean PAX2 expression score compared to regression patients, a risk threshold was

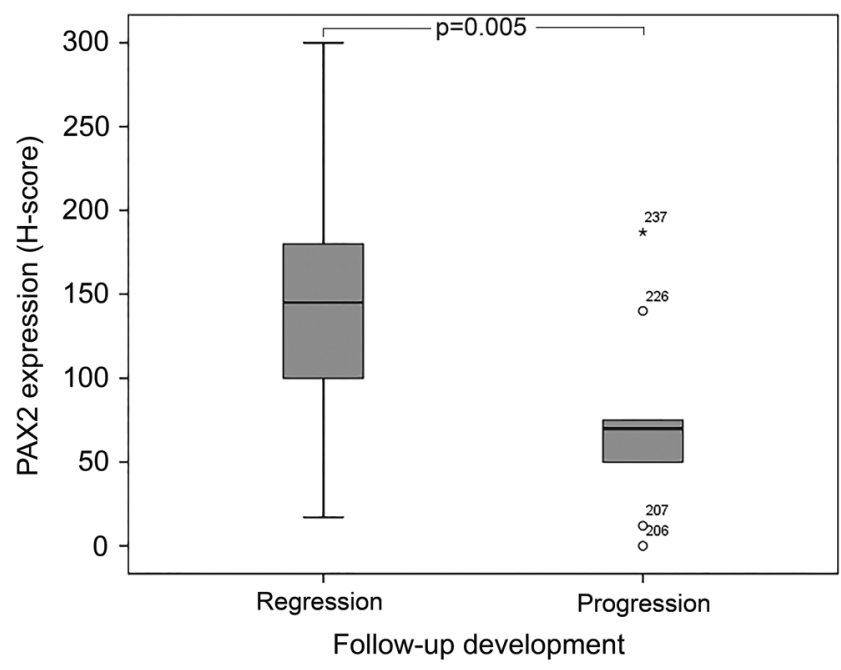

Figure 3

PAX2 expression of EIN patients that demonstrated either progression $(n=10)$ or regression $(n=26)$ in the follow-up. Patients with an initial EIN diagnosis where progression was observed in the follow-up had a significantly lower PAX2 expression score than those that regressed (independent samples $t$-test, $P=0.005$ ).
Table 3 Mean age, follow-up time, PAX2 score and PTEN status according to follow-up status in each diagnostic category.

\begin{tabular}{|c|c|c|}
\hline Initial diagnosis & Follow-up diagnosis & \\
\hline $\mathrm{PE}^{*}(N=75)$ & No change & Progression \\
\hline$N(62)$ & 62 & 0 \\
\hline Age & $37(25-50)$ & \\
\hline $\begin{array}{l}\text { Follow-up time } \\
\text { (months) }\end{array}$ & $115(6-202)$ & \\
\hline PAX2 score & $155(0-245)$ & \\
\hline $\begin{array}{l}\text { PTEN status } \\
(N=58)\end{array}$ & $\begin{array}{l}83 \% \text { pos }(49 / 59) \\
17 \% \text { neg }(10 / 59)\end{array}$ & \\
\hline $\operatorname{EIN}^{+}(N=36)$ & Regression & Progression \\
\hline$N(36)$ & 26 & 10 \\
\hline Age & $42(24-59)^{\S}$ & $57(38-87)^{\S}$ \\
\hline $\begin{array}{l}\text { Follow-up time } \\
\text { (months) }\end{array}$ & $150(16-275)^{\S}$ & $44(13-114)^{\S}$ \\
\hline PAX2 score & $142(17-300)^{\S}$ & $73(0-187)^{\S}$ \\
\hline PTEN score $(N=35)$ & $\begin{array}{l}80 \% \operatorname{pos}(20 / 25) \\
20 \% \text { neg }(5 / 25)\end{array}$ & $\begin{array}{l}60 \% \text { pos }(6 / 10) \\
40 \% \text { neg }(4 / 10)\end{array}$ \\
\hline D-score $(N=35)$ & $1.8^{\S}(-1.6$ to 4.8$)$ & $0.12^{\S}(-2.8$ to 2.4$)$ \\
\hline $\operatorname{EEC}^{\ddagger}(N=183)$ & Regression & Progression \\
\hline$N(178)$ & 158 & 20 \\
\hline Age & $66(32-92)$ & $70(43-86)$ \\
\hline $\begin{array}{l}\text { Follow-up time } \\
\text { (months) }\end{array}$ & $141(6-310)^{\S}$ & $46(6-196)^{\S}$ \\
\hline PAX2 score & $56(0-215)$ & $53(0-177)$ \\
\hline $\begin{array}{l}\text { PTEN score } \\
(N=172)\end{array}$ & $\begin{array}{l}35 \% \text { pos }(53 / 152) \\
65 \% \text { neg }(99 / 152)\end{array}$ & $\begin{array}{l}40 \% \text { pos }(8 / 20) \\
60 \% \text { neg }(12 / 20)\end{array}$ \\
\hline D-score $(N=160)$ & $-1.17(-4.2$ to 0.9$)$ & $-1.5(-3.3$ to 0.8$)$ \\
\hline
\end{tabular}

*Proliferative endometrium; †endometrial intraepithelial neoplasia; ‡endometrioid endometrial carcinoma; §significant: independent samples $t$-test, $P<0.05$.

established for PAX2 in order to group patients into progression risk categories. For the purpose of this study, a threshold score of 75 was used to identify patients with low risk of progression: PAX2 $>75$, and patients with a high risk of progression: PAX $2 \leq 75$. This threshold was based on box plot analysis where $75 \%$ of all EIN patients that progressed had a PAX2 score equal to or below 75 (Fig. 3) and where ROC analysis revealed that specificity decreased beyond this threshold (data not shown). The threshold defined $4 \%$ of PE cases as high-risk $(n=75), 31 \%$ of EIN $(n=36)$ and $68 \%$ of EEC $(n=183)$. None of the patients with PE developed EIN or EEC. For patients with an initial EIN diagnosis, follow-up revealed that $73 \%(8 / 11)$ of patients with a high-risk PAX2 score progressed and only $8 \%(2 / 25)$ of patients with a low-risk PAX2 score progressed (Table 4). To note, at final follow-up, no EIN patients were dead due to EEC-related or unrelated causes $(0 / 36)$. For patients in the EEC category where follow-up was available $(n=178), 10 \%(12 / 121)$ of EEC patients with a highrisk score demonstrated progression whilst $14 \%(8 / 57)$ 
of EEC patients with a low-risk score demonstrated progression (Table 4).

For risk analysis according to PTEN status, we defined PTEN negative as high risk of progression and PTEN positive as low risk of progression due to the observation that PTEN loss occurs at a higher percentage in EEC in comparison to PE and EIN. For patients with an initial diagnosis of EIN, we observed progression in 44\% (4/9) of high-risk cases and 23\% (6/26) of low-risk cases (Table 4). Whereas for patients with an initial diagnosis of EEC, we observed progression in 13\% (3/61) of high-risk cases and $11 \%(12 / 111)$ of low-risk cases (Table 4$)$.

To assess whether the PAX2 threshold or PTEN status had any significant prognostic value in EIN or EEC, we utilised Kaplan-Meier curve analysis to assess progression-free survival. Analysis revealed that highrisk EIN patients classified by PAX2 expression score had a significant lower progression-free survival than low-risk patients (Fig. 4). However, for patients with EEC, there was no difference in progression-free survival between patients with PAX2 low-risk or highrisk status (Table 4). Analysis of PTEN status showed no significant difference in progression-free survival for EIN and EEC patients (Table 4). We also analysed the PAX2 risk threshold in combination with PTEN status and D-score but no additional prognostic value (from PTEN) was observed in either EIN or EEC (Table 4).

Table 4 Overview of the proportion of patients that demonstrated disease progression in either EIN or EEC according to risk classification defined by PAX2 score, PTEN status and/or D-score.

\begin{tabular}{|c|c|c|c|c|c|}
\hline Biomarker & Risk status & $\begin{array}{l}\text { Percent EIN* patients } \\
\text { that progressed }{ }^{+}\end{array}$ & $\begin{array}{c}\text { Survival analysis } \\
\text { (Kaplan-Meier, log rank) }\end{array}$ & $\begin{array}{l}\text { Percent EEC }{ }^{\ddagger} \text { patients } \\
\text { that progressed } \$\end{array}$ & $\begin{array}{c}\text { Survival analysis } \\
\text { (Kaplan-Meier, log rank) }\end{array}$ \\
\hline \multicolumn{6}{|l|}{ PAX2 } \\
\hline$\leq 75$ & High & $73 \%(8 / 11)$ & $P=0.001$ & $10 \%(12 / 121)$ & $P=0.473$ \\
\hline$>75$ & Low & $8 \%(2 / 25)$ & & $14 \%(8 / 57)$ & \\
\hline \multicolumn{6}{|l|}{ PTEN } \\
\hline Negative & High & $44 \%(4 / 9)$ & $P=0.268$ & $13 \%(8 / 61)$ & $P=0.719$ \\
\hline Positive & Low & $23 \%(6 / 26)$ & & $11 \%(12 / 111)$ & \\
\hline \multicolumn{6}{|l|}{ D-score } \\
\hline$<1$ & High & $50 \%(6 / 12)$ & $P=0.094$ & N/A & \\
\hline$\geq 1$ & Low & $17 \%(4 / 23)$ & & & \\
\hline \multicolumn{6}{|c|}{ PAX2 PTEN in four risk groups } \\
\hline $\begin{array}{l}\leq 75 \\
\text { Negative }\end{array}$ & High & $67 \%(4 / 6)$ & $P=0.012$ & $11 \%(9 / 81)$ & $P=0.603$ \\
\hline $\begin{array}{l}\leq 75 \\
\text { Positive }\end{array}$ & Uncertain & $80 \%(4 / 5)$ & & $8 \%(3 / 36)$ & \\
\hline $\begin{array}{l}>75 \\
\text { Negative }\end{array}$ & Uncertain & $0 \%(0 / 3)$ & & $1 \%(3 / 30)$ & \\
\hline $\begin{array}{l}>75 \\
\text { Positive }\end{array}$ & Low & $10 \%(2 / 21)$ & & $20 \%(5 / 25)$ & \\
\hline \multicolumn{6}{|c|}{ PAX2 D-score in four risk groups } \\
\hline $\begin{array}{l}\leq 75 \\
<1\end{array}$ & High & $100 \%(5 / 5)$ & $P=0.009$ & N/A & \\
\hline $\begin{array}{l}\leq 75 \\
\geq 1\end{array}$ & Uncertain & $50 \%(3 / 6)$ & & & \\
\hline $\begin{array}{l}>75 \\
<1\end{array}$ & Uncertain & $17 \%(1 / 6)$ & & & \\
\hline $\begin{array}{l}>75 \\
\geq 1\end{array}$ & Low & $6 \%(1 / 18)$ & & & \\
\hline \multicolumn{6}{|c|}{ PTEN and D-score in four risk groups } \\
\hline $\begin{array}{l}\text { Negative } \\
<1\end{array}$ & High & $67 \%(4 / 6)$ & $P=0.388$ & N/A & \\
\hline $\begin{array}{l}\text { Negative } \\
\geq 1\end{array}$ & Uncertain & $0 \%(0 / 3)$ & & & \\
\hline $\begin{array}{l}\text { Positive } \\
<1\end{array}$ & Uncertain & $33 \%(2 / 6)$ & & & \\
\hline $\begin{array}{l}\text { Positive } \\
\geq 1\end{array}$ & Low & $20 \%(4 / 20)$ & & & \\
\hline
\end{tabular}

*Endometrial intraepithelial neoplasia; †progression event for EIN was defined as a diagnosis of persistent EIN with more poorly differentiated glands or diagnosis of EEC in the follow-up; ${ }^{*}$ endometrioid endometrial carcinoma; sprogression event for EEC was defined as higher stage EEC or death due to EEC. 


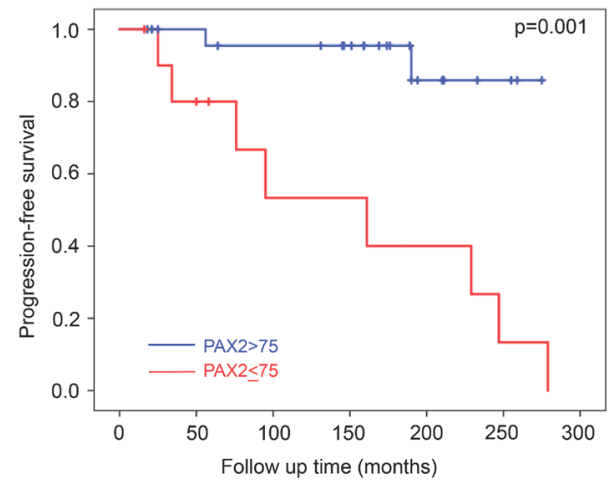

Figure 4

Long-term progression-free survival curve of patients initially diagnosed with EIN according to risk status determined by PAX2 expression score. For EIN samples that were defined as high-risk, where PAX2 $\leq 75$ ( $n=11$, red) patients had a significantly lower progression-free survival than low-risk patients, where PAX $>75$ ( $n=25$, blue) (Kaplan-Meier log rank, $P=0.001)$. A full colour version of this figure is available at https://doi. org/10.1530/ERC-18-0106

\section{Discussion}

In this study, we have used strictly optimised and standardised immunohistochemistry to observe expression patterns of PTEN and PAX2 in PE, EIN and EEC. A higher proportion of PTEN-negative cases were observed in EEC than in EIN and PE. Furthermore, PAX2 expression decreased progressively from PE to EIN to EEC.

PTEN loss was detected in $11 \%$ of PE samples, which is similar to results found in three separate studies (Feng et al. 2012, Lee et al. 2012, Yang et al. 2015). In EEC, PTEN loss occurred in $66 \%$ of samples which is similar to findings in literature where loss has been observed to range from 55 to $70.2 \%$ (Kanamori et al. 2002, Uegaki et al. 2005, Mackay et al. 2010, Monte et al. 2010, Feng et al. 2012, Lee et al. 2012, Yang et al. 2015). For 11\% of EIN cases, PTEN loss was observed, equal to that of $\mathrm{PE}$, which is far lower than what has been observed in the literature with $44 \%$ and $63 \%$ observed in two separate studies where the EIN classification was used (Mutter et al. 2001, Monte et al. 2010), and in studies where the WHO94 scheme has been used, PTEN loss has been observed at $42.9-71 \%$ in the category atypical hyperplasia (Lacey et al. 2008, Feng et al. 2012, Lee et al. 2012). The discrepancy here between our observation and the literature could be due to our relatively small sample size for EIN.

In addition, no association between PTEN status and risk of progression in patients diagnosed with EIN or EEC was found. Previously, PTEN status has been shown to have a low predictive value in predicting clinical outcome of EIN, however, in combination with D-score, its prognostic value is increased (Baak et al. 2005b). It is important to note that the sample number of EIN patients that progressed was very small in both their and our studies $(n=7)$. These findings would need to be validated in a larger population. Supporting our preliminary findings, Lacey et al. (2008) demonstrated in a large sample group that PTEN status was not associated with progression risk of endometrial hyperplasia.

With regards to PTEN status in EEC, several studies report that there is an association with PTEN status and progression in EEC but even here there are discrepancies. Some studies state that a PTEN-positive status is associated with a favourable prognosis (Kanamori et al. 2002, Uegaki et al. 2005) and that with higher stages of EEC, PTEN loss increases (Daniilidou et al. 2013), but two studies suggest that a PTEN-negative status is associated with a favourable prognosis (Mackay et al. 2010, AkiyamaAbe et al. 2013). The present study found no association between PTEN status and progression-free survival in EEC or any difference between PTEN status and FIGO stage. In combination, our findings and those of the literature makes PTEN a challenging prognostic biomarker for both EIN and EEC.

In the present study, a progressive decrease in PAX2 expression from PE to EIN to EEC was observed, which is also demonstrated in two separate studies (Monte et al. 2010, Allison et al. 2012). This suggests that loss of PAX2 expression is an early event of endometrial carcinogenesis and clonal expansion of these glands. For the purposes of this study, the H-score method was used to assess PAX2 expression in endometrial samples, whereas other studies have scored based on the percentage of PAX2 null glands in a sample (Monte et al. 2010) or grouped patients based on complete loss ( $0 \%$ cells staining), partial loss (1-75\% cells staining) or minimal to no loss $(76-100 \%$ cells staining) of PAX2 (Allison et al. 2012) or the number of positive cells per 1000 epithelial cells or tumour cells for EEC cases (Kahraman et al. 2012). Of interest, Kahraman et al. (2012) reported an increase in PAX2 expression with increasing severity of diagnosis despite the use of two different scoring methods including one employed by Monte et al. (2010) who had previously reported the opposite observation akin to our findings. Discrepancies here could be due to different immunohistochemistry methods. Whilst Monte et al. (2010) has used the same antibody utilised in the present study, Kahraman et al. (2012) made use of another. On closer comparison 
Kahraman et al. (2012) observed PAX2 staining in the stroma, an observation neither we nor several other studies (Monte et al. 2010, Allison et al. 2012, Joiner et al. 2015, Ørbo et al. 2015) have observed.

In this study, we present a method and threshold that could prove promising for identification of high-risk EIN lesions that will progress to EEC. EIN lesions with a PAX2 expression H-score below or equal to 75 (high risk) had significantly lower progression-free survival than those with an $\mathrm{H}$-score above 75 (low risk). Furthermore, more EIN patients with high-risk PAX2 showed progression (73\%; 8/11) compared to low-risk patients $(8 \% ; 2 / 25)$. This is the first time, to our knowledge, that PAX2 expression has been associated with progression in EIN in a population with long-term follow-up. We acknowledge that this study uses a small EIN sample number and therefore would need to be validated in a large patient cohort; however, we would like to emphasise that use of PAX2 for risk categorisation of EIN holds promise and should be investigated further. To be noted, a previous study demonstrated no association with PAX2 expression and progression of endometrial hyperplasia, however, this study had a very short follow-up of 2-6 months (Upson et al. 2012). It is recognised that endometrial carcinogenesis is a long process and development of EEC from normal endometrium can take 30-40 years where progression of EIN to EEC can take as long as 10 years. Although our study is limited by the small EIN sample size, the follow-up of these patients is long. Unlike for EIN, the PAX2 expression threshold was not prognostic in EEC, where patients with a high-risk score progressed similarly to those with a low-risk score (13\% vs $11 \%)$. Furthermore, no additional benefit was demonstrated of using PTEN status in combination with PAX2 to determine progression in either EIN or EEC.

A challenge in comparing literature, and what could account for the variations seen between our observations and previous studies, is the use of different immunohistochemistry methods, observed staining patterns and scoring protocols. Some have observed staining in both stroma and glands whilst others only observe nuclear staining of the endometrial glands. Interpretation of immunostained tissue can be a significant limiting factor for accurate diagnostic and prognostic evaluation. By utilising digital image analysis, this variability and error due to subjective interpretation of staining patterns can be reduced. Immunostaining of PAX2 has been shown to be technically robust and its distinct nuclear staining is not difficult to interpret (Monte et al. 2010, Quick et al. 2012, Ørbo et al. 2015).
In the current study, reproducibility of scoring was high. A benefit of PAX2 staining is adjacent normal endometrial tissue functions well as an internal control (Quick et al. 2012).

In conclusion, decreased PAX2 expression is associated with clinical progression of EIN lesions. Thus, evaluation of PAX2 expression in EIN lesions could have significant impact on treatment decisions. To be able to distinguish between lesions that ought to be treated differently could significantly reduce the number of patients that are over and under treated and further investigation is therefore warranted.

\section{Declaration of interest}

The authors declare that there is no conflict of interest that could be perceived as prejudicing the impartiality of the research reported.

\section{Funding}

Anita Steinbakk received funding from Helse Vest, grant \#911268.

\section{Author contribution statement}

$J B$ and $E A M ~ J$ designed the study. E V, E A M J, J B, I S, E G performed the research. A E V, A S, E A M J, I S, E G, B V D, E R, J B contributed to data acquisition and quality control. A E V, J B, E A M J, E R analysed the data. E R wrote the manuscript. All authors provided feedback.

\section{Acknowledgements}

The authors would like to thank Marit Nordhus for her hard work and excellent technical assistance.

\section{References}

Akiyama-Abe A, Minaguchi T, Nakamura Y, Michikami H, Shikama A, Nakao S, Sakurai M, Ochi H, Onuki M, Matsumoto K, et al. 2013 Loss of PTEN expression is an independent predictor of favourable survival in endometrial carcinomas. British Journal of Cancer 109 1703-1710. (https://doi.org/10.1038/bjc.2013.455)

Allison KH, Upson K, Reed SD, Jordan CD, Newton KM, Doherty J, Swisher EM \& Garcia RL 2012 PAX2 loss by immunohistochemistry occurs early and often in endometrial hyperplasia. International Journal of Gynecological Pathology 31 151-159. (https://doi. org/10.1097/PGP.0b013e318226b376)

Baak JPA, Ørbo A, Van Diest PJ, Jiwa M, de Bruin P, Broeckaert M, Snijders W, Boodt PJ, Fons G, Burger C, et al. 2001 Prospective multicenter evaluation of the mophometric D-score for prediction of the outcome of endometrial hyperplasias. American Journal of Surgical Pathology 25 930-935.

Baak JP, Mutter GL, Robboy S, van Diest PJ, Uyterlinde AM, Ørbo A, Palazzo J, Fianne B, Løvslett K, Burger C, et al. 2005a The molecular genetics and morphometry-based endometrial intraepithelial neoplasia classification system predicts disease progression in endometrial hyperplasia more accurately than the 1994 World (c) 2018 Society for Endocrinology Published by Bioscientifica Ltd. Printed in Great Britain 
Health Organization Classification System. Cancer 103 2304-2312. (https://doi.org/10.1002/cncr.21058)

Baak JPA, van Diermen B, Steinbakk A, Janssen E, Skaland I, Mutter GL, Fiane B \& Løvslett K 2005b Lack of PTEN expression in endometrial intraepithelial neoplasia is correlated with cancer progression. Human Pathology 36 555-561. (https://doi.org/10.1016/j.humpath.2005.02.018)

Bergeron C, Nogales FF, Masseroli M, Abeler V, Duvillard P, MüllerHolzner E, Pickartz H \& Wells M 1999 A multicentric European study testing the reproducibility of the WHO classification of endometrial hyperplasia with a proposal of a simplified working classification for biopsy and curettage specimens. American Journal of Surgical Pathology 23 1102-1108. (https://doi.org/10.1097/00000478199909000-00014)

Bokhman JV 1983 Two pathogenetic types of endometrial carcinoma. Gynecologic Oncology 15 10-17. (https://doi.org/10.1016/00908258(83)90111-7)

Daniilidou K, Frangou-Plemenou M, Grammatikakis J, Grigoriou O, Vitoratos N \& Kondi-Pafiti A 2013 Prognostic significance and diagnostic value of PTEN and p53 expression in endometrial carcinoma. A retrospective clinicopathological and immunohistochemical study. Journal of the Balkan Union of Oncology 18 195-201.

Feng Z-Z, Chen J-W, Yang Z-R, Lu G-Z \& Cai Z-G 2012 Expression of PTTG1 and PTEN in endometrial carcinoma: correlation with tumorigenesis and progression. Medical Oncology 29 304-310. (https://doi.org/10.1007/s12032-010-9775-x)

Hecht JL, Ince TA, Baak JP, Baker HE, Ogden MW \& Mutter GL 2005 Prediction of endometrial carcinoma by subjective endometrial intraepithelial neoplasia diagnosis. Modern Pathology 18 324-330. (https://doi.org/10.1038/modpathol.3800328)

Joiner AK, Quick CM \& Jeffus SK 2015 PAX2 expression in simultaneously diagnosed WHO and EIN classification systems. International Journal of Gynecological Pathology 34 40-46. (https://doi. org/10.1097/PGP.0000000000000185)

Kahraman K, Kiremitci S, Taskin S, Kankaya D, Sertcelik A \& Ortac F 2012 Expression pattern of PAX2 in hyperplastic and malignant endometrium. Archives of Gynecology and Obstetrics 286 173-178. (https://doi.org/10.1007/s00404-012-2236-3)

Kanamori Y, Kigawa J, Itamochi H, Sultana H, Suzuki M, Ohwada M, Kamura T, Sugiyama T, Kikuchi Y \& Kita T 2002 PTEN expression is associated with prognosis for patients with advanced endometrial carcinoma undergoing postoperative chemotherapy. International Journal of Cancer 100 686-689. (https://doi. org/10.1002/ijc.10542)

Kendall BS, Ronnett BM, Isacson C, Cho KR, Hedrick L, Diener-West M \& Kurman RJ 1998 Reproducibility of the diagnosis of endometrial hyperplasia, atypical hyperplasia, and well-differentiated carcinoma. American Journal of Surgical Pathology 22 1012-1019.

Kurman RJ, Carcangiu ML, Herrington CS \& Young RH 2014 WHO Classification of Tumours of Female Reproductive Organs. Lyon, France: International Agency for Research on Cancer.

Kurose K, Zhou X-P, Araki T, Cannistra SA, Maher ER \& Eng C 2001 Frequent loss of PTEN expression is linked to elevated phosphorylated Akt levels, but not associated with p27 and cyclin D1 expression, in primary epithelial ovarian carcinomas. American Journal of Pathology 158 2097-2106. (https://doi.org/10.1016/S00029440(10)64681-0)

Lacey JV, Mutter GL, Ronnett BM, Ioffe OB, Duggan MA, Rush BB, Glass AG, Richesson DA, Chatterjee N, Langholz B, et al. 2008 PTEN expression in endometrial biopsies as a marker of progression to endometrial carcinoma. Cancer Research 68 6014-6020. (https://doi. org/10.1158/0008-5472.CAN-08-1154)

Lee H, Choi HJ, Kang CS, Lee HJ, Lee WS \& Park CS 2012 Expression of miRNAs and PTEN in endometrial specimens ranging from histologically normal to hyperplasia and endometrial adenocarcinoma. Modern Pathology 25 1508-1515. (https://doi. org/10.1038/modpathol.2012.111)

Mackay HJ, Gallinger S, Tsao MS, McLachlin CM, Tu D, Keiser K, Eisenhauer EA \& Oza AM 2010 Prognostic value of microsatellite instability (MSI) and PTEN expression in women with endometrial cancer: results from studies of the NCIC Clinical Trials Group (NCIC CTG). European Journal of Cancer 46 1365-1373. (https://doi. org/10.1016/j.ejca.2010.02.031)

Monte NM, Webster KA, Neuberg D, Dressler GR \& Mutter GL 2010 Joint loss of PAX2 and PTEN expression in endometrial precancers and cancer. Cancer Research 70 6225-6232. (https://doi. org/10.1158/0008-5472.CAN-10-0149)

Mutter GL \& Group TIENW 2000 Endometrial intraepithelial neoplasia (EIN): will it bring order to chaos? Gynecologic Oncology 76 287-290. (https://doi.org/10.1006/gyno.1999.5580)

Mutter GL, Lin M-C, Fitzgerald JT, Kum JB, Baak J, Lees JA, Weng L-P \& Eng C 2000 Altered PTEN expression as a diagnostic marker for the earliest endometrial precancers. Journal of the National Cancer Institute 92 924-930. (https://doi.org/10.1093/jnci/92.11.924)

Mutter GL, Ince TA, Baak JP, Kust GA, Zhou X-P \& Eng C 2001 Molecular identification of latent precancers in histologically normal endometrium. Cancer Research 61 4311-4314.

Ørbo A, Arnes M, Lyså LM \& Straume B 2015 Expression of PAX2 and PTEN correlates to therapy response in endometrial hyperplasia. Anticancer Research 35 6401-6409.

Pavlakis K, Messini I, Vrekoussis T, Panoskaltsis T, Chrissanthakis D, Yiannou P \& Stathopoulos EN 2010 PTEN-loss and nuclear atypia of EIN in endometrial biopsies can predict the existence of a concurrent endometrial carcinoma. Gynecologic Oncology 119 516-519. (https://doi.org/10.1016/j.ygyno.2010.08.023)

Quick CM, Laury AR, Monte NM \& Mutter GL 2012 Utility of PAX2 as a marker for diagnosis of endometrial intraepithelial neoplasia. American Journal of Clinical Pathology 138 678-684. (https://doi. org/10.1309/AJCP8OMLT7KDWLMF)

Shang Y 2007 Hormones and cancer. Cell Research 17 277. (https://doi. org/10.1038/cr.2007.26)

Skov B, Broholm H, Engel U, Franzmann M-B, Nielsen A, Lauritzen A \& Skov T 1997 Comparison of the reproducibility of the WHO classifications of 1975 and 1994 of endometrial hyperplasia. International Journal of Gynecological Pathology 16 33-37. (https://doi. org/10.1097/00004347-199701000-00006)

Steinbakk A, Skaland I, Gudlaugsson E, Janssen EA, Kjellevold KH, Klos J, Løvslett K, Fiane B \& Baak JP 2009 The prognostic value of molecular biomarkers in tissue removed by curettage from FIGO stage 1 and 2 endometrioid type endometrial cancer. American Journal of Obstetrics and Gynecology 200 78.e71-78.e78. (https://doi. org/10.1016/j.ajog.2008.07.020)

Strissel PL, Ellmann S, Loprich E, Thiel F, Fasching PA, Stiegler E, Hartmann A, Beckmann MW \& Strick R 2008 Early aberrant insulin-like growth factor signaling in the progression to endometrial carcinoma is augmented by tamoxifen. International Journal of Cancer 123 2871-2879. (https://doi.org/10.1002/ ijc.23900)

Uegaki K, Kanamori Y, Kigawa J, Kawaguchi W, Kaneko R, Naniwa J, Takahashi M, Shimada M, Oishi T, Itamochi H, et al. 2005 PTENpositive and phosphorylated-Akt-negative expression is a predictor of survival for patients with advanced endometrial carcinoma. Oncology Reports 14 389-392.

Upson K, Allison KH, Reed SD, Jordan CD, Newton KM, Swisher EM, Doherty JA \& Garcia RL 2012 Biomarkers of progestin therapy resistance and endometrial hyperplasia progression. American Journal of Obstetrics and Gynecology 207 36.e31-36.e38. (https://doi. org/10.1016/j.ajog.2012.05.012)
(2) 2018 Society for Endocrinology Published by Bioscientifica Ltd. Printed in Great Britain 
Usubutun A, Mutter GL, Saglam A, Dolgun A, Ozkan EA, Ince T, Akyol A, Bulbul HD, Calay Z, Eren F, et al. 2012 Reproducibility of endometrial intraepithelial neoplasia diagnosis is good, but influenced by the diagnostic style of pathologists. Modern Pathology 25 877-884. (https://doi.org/10.1038/ modpathol.2011.220)

Wu H, Chen Y, Liang J, Shi B, Wu G, Zhang Y, Wang D, Li R, Yi X \& Zhang H 2005 Hypomethylation-linked activation of PAX2 mediates tamoxifen-stimulated endometrial carcinogenesis. Nature $\mathbf{4 3 8}$ 981-987. (https://doi.org/10.1038/nature04225)

Yang HP, Meeker A, Guido R, Gunter MJ, Huang GS, Luhn P, d'Ambrosio L, Wentzensen N \& Sherman ME 2015 PTEN expression in benign human endometrial tissue and cancer in relation to endometrial cancer risk factors. Cancer Causes and Control 26 1729-1736. (https://doi.org/10.1007/s10552-0150666-5)

Received in final form 5 June 2018

Accepted 10 July 2018
() 2018 Society for Endocrinology Published by Bioscientifica Ltd. Printed in Great Britain 